\title{
Geosynchronous Network Grid Addressing for Integrated Space-Terrestrial Networks
}

\author{
Gao Zheng, Ning Wang, Rahim Tafazolli \\ 5GIC, University of Surrey, Guildford, Surrey, UK \\ $\{$ g.zheng, n.wang, r.tafazolli\}@Surrey.ac.uk
}

\author{
Xinpeng Wei \\ Huawei Technologies Co.,Ltd. Beijing, China \\ weixinpeng@huawei.com
}

\begin{abstract}
The launch of the StarLink Project has recently stimulated a new wave of research on integrating Low Earth Orbit (LEO) satellite networks with the terrestrial Internet infrastructure. In this context, one distinct technical challenge to be tackled is the frequent topology change caused by the constellation behaviour of LEO satellites. Frequent change of the peering IP connection between the space and terrestrial Autonomous Systems (ASes) inevitably disrupts the Border Gateway Protocol (BGP) routing stability at the network boundaries which can be further propagated into the internal routing infrastructures within ASes. To tackle this problem, we introduce the Geosynchronous Network Grid Addressing (GNGA) scheme by decoupling IP addresses from physical network elements such as a LEO satellite. Specifically, according to the density of LEO satellites on the orbits, the IP addresses are allocated to a number of stationary "grids" in the sky and dynamically bound to the interfaces of the specific satellites moving into the grids along time. Such a scheme allows static peering connection between a terrestrial BGP speaker and a fixed external BGP (e-BGP) peer in the space, and hence is able to circumvent the exposure of routing disruptions to the legacy terrestrial ASes. This work-in-progress specifically addresses a number of fundamental technical issues pertaining to the design of the GNGA scheme.

Index Terms-Space-Terrestrial network integration, Networking with LEO Satellites, Satellite Internet, Routing, Dynamictopology networking.
\end{abstract}

\section{INTRODUCTION}

In essence, space-terrestrial network integration utilizes the Internet technologies to build a global-covered, fast, reliable heterogeneous network which consists of Geostationary Earth Orbit (GEO), Medium Earth Orbit (MEO), Low Earth Orbit (LEO) satellites as well as the existing terrestrial networks. In the last few years, US, China and EU have successively brought space-terrestrial network integration into their own international strategic plannings [1]. Especially the research interest on LEO satellite integration has dramatically increased recently, since SpaceX has continuously launched its LEO satellites with authorizations granted from the US Federal Communications Committee, meaning that the era of spaceterrestrial integrated Internet is coming. As pointed out in [2], with the LEO satellite constellations the capability of terrestrial network in terms of long-distance transmission can be greatly enhanced based on the fact that optical signal is travailing $47 \%$ faster in vacuum than in glass. Meanwhile, the LEO satellite network provides ubiquitous access ability in addition to the terrestrial network which is particularly significant

978-1-7281-6992-7/20/\$31.00 @2020 IEEE for scenarios such as disaster relief, aircraft/transoceanic communication. Moreover, LEO satellite network is also promising in providing transit services across terrestrial ASes [2]. If further considering the great success achieved by today's Internet technologies, it is confident to foretell that the demand for natural in-space IP framework support is inevitable, and by achieving it, future network application scenarios such as IoT in the space, caching/edge computing from the sky become available.

However, realizing these exciting prospects requires addressing new challenges. Due to the mutual independent developments in the early stage, current terrestrial network infrastructure has a fundamental different routing suite compared to its space counterpart. Especially the originally designed interdomain routing protocols are tailored for fixed networks whose design mainly considers the accuracy of routing reachability and reckons without the impact caused by topology dynamics. Take the widespread BGP as an example, any topology change will trigger the routing calculation and it takes several minutes to converge even for a small-scale network [3]. This is tolerable in the terrestrial network since the topology of the terrestrial network is relatively stable which barely changes within hours or even days [4]. But for LEO satellite networks, as the constellation moves constantly, the physical connections between the space and terrestrial network will change in much shorter time scales [5]. Thus, naively adopting BGP in the space-terrestrial network integration will end up with issues such as unstable neighborhood, over-frequency routing updates and slow convergence time, which can cause serious impact to the existing terrestrial network system. Consequently, how to protect the terrestrial network from the LEO satellite constellation's high topology dynamics is one of the key challenges in space-terrestrial network integration research.

To tackle this issue, in this work-in-process we design the integration by introducing a novel scheme, namely, the Geosynchronous Network Grid Addressing (GNGA). Unlike classic integration designs that associate IP addresses with satellites, with GNGA we decouple the IP address from the satellites and bind the addresses based on fixed geography locations. In this way, the GNGA scheme is able to hide the constellation movement from the network layer thereby bypass issues caused by the topology dynamics. More specifically, depending on the density of launched LEO satellites along the orbits, the space above the terrestrial Internet can 
be partitioned into fixed logical network grids, each being identified with fixed IP addresses. From the viewpoint of terrestrial network infrastructures, their e-BGP peers are the fixed logical IP addresses covering the specific space grids, while such addresses are dynamically instantiated by the LEO satellites sequentially entering the grids based on a predictable manner. Overall, the major contributions of this work are the following:

- We introduce a novel Space-Terrestrial network integration scheme called the Geosynchronous Network Grid Addressing, with which the topology dynamic issue caused by LEO satellite constellation is circumvented.

- The designed integration scheme causes little impact to the existing terrestrial network system and is easy to deploy.

\section{BACKGROUND}

Based on the role of the satellite network, recent proposed space-terrestrial network integration schemes can be roughly classified as the following categories: 1) Satellite as an independent network; 2) Satellite as the access network; 3) Satellite as the transit network.

In the first category, the satellite network and the terrestrial network are different networking systems. As an independent system, routing inside a satellite constellation is a well-studied topic and works like [6]-[9] have provided high-performance routing schemes for the dynamic satellite networks. However, for this kind of integration scheme, when terrestrial data packets transit the space-terrestrial network border, additional headers will be applied to the original IP header for routing in space networks.

With the development of 5G network technology, LEO satellites are utilized to extend the access coverage for the terrestrial network. For example, [10] and [11] have provided the access technologies to integrate the LEO satellites into the Internet and the $5 \mathrm{G}$ core network respectively. However, these works mainly focus on the access technology itself lacking a comprehensive view on the communication and data transmission needs to rely on the terrestrial network, which even applies to the scenario where the two communication ends are with the same satellite. Hence, the flexibility of satellite transmission in these integration schemes needs to be improved.

A number of recent works have proposed to use the satellite network as the transit network. For example, a space-terrestrial dual-backbone architecture is proposed in [1], The authors discussed several key technical challenges associated with the architecture including routing, interworking network protocols, transmission, however, the concrete solution is not provided. [12] utilizes LEO satellites to enhance to the long-distance transmission ability for the terrestrial network, however, the work is based on the path-aware network architecture [13] which is different from the mainstream of hop-by-hop IP routing philosophy.

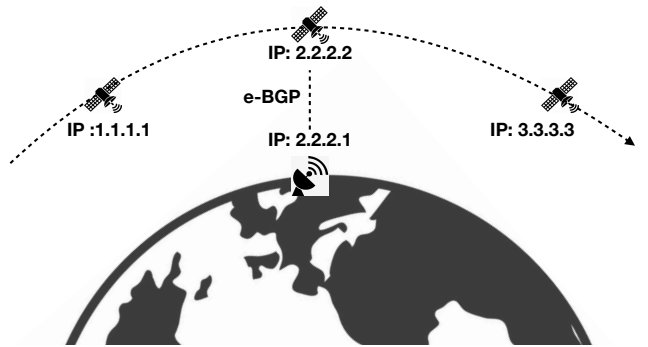

Fig. 1. Naive Scheme.

\section{SPACE-TERRESTRIAL NETWORK INTEGRATION SCHEME WITH GNGA}

In this section, we present in detail the designed integration scheme. Generally speaking, a space-terrestrial integrated network is constructed by two components: the space segment and the terrestrial segment. The terrestrial segment typically consists of a large number of fixed ASes or domains, while the space segment can be also viewed as a separate AS operated by a satellite operator. In this case, the interconnection between the space and terrestrial segment is typically through e-BGP. As will be detailed in Section III-A, with the traditional IP addressing system, it is expected that e-BGP between the two segments will see frequent changes of peering IP addresses which will lead to significant routing disruptions at both the network boundaries and inside affected domains due to the propagation into internal BGP (i-BGP).

Our design goal is to seamlessly integrate the space and terrestrial networks based on a unified common IP infrastructure, but without introducing severe routing stabilities caused by the LEO satellite constellation behaviours, especially considering the legacy routing infrastructure on the terrestrial network side. To tackle the space-terrestrial mutual topology dynamics, our strategy is to make the space segment geosynchronous and thus we introduce the GNGA. With GNGA, we create a number of geosynchronous grids along the LEO satellite orbits to act as virtual IP routers at fixed location grids. We require the LEO satellites to consecutively undertake the control and data plane functions when them move across the geosynchronous grids. We further decouple the IP address from the satellites and assign it to the geosynchronous grids. Thereby, from the perspective of the network layer, the space segment network is stable. Meanwhile, in order to support GNGA scheme, we also present the design of an auxiliary Medium Access Control (MAC) layer mechanism to enable the mapping operation from layer 3 addressing to the reachability of a grid.

\section{A. The Satellite-terrestrial routing stability problem}

To help better understanding the problem, we provide here a simple illustration. Figure 1 shows a naive scenario where BGP is directly applied in the integration. As depicted, each LEO satellite is assigned with a dedicated fixed IP address on its downlink interface towards the ground stations. In order to communicate with the space segment, a ground station needs 


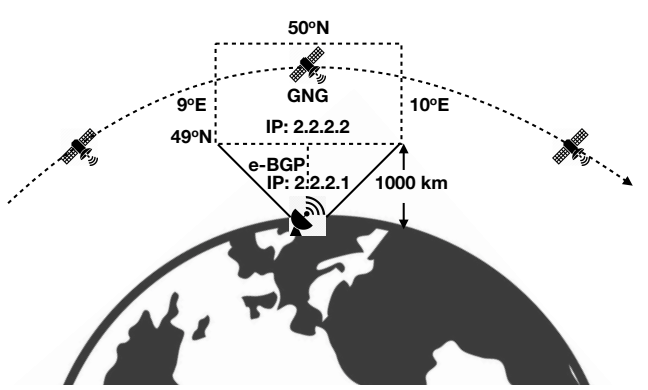

Fig. 2. Geosynchronous Network Grid Addressing.

to maintain the BGP peers using the external peering satellite IP addresses. However, since the LEO satellites move at a high velocity relative to the ground, it is expected that when a satellite moves far away, the ground station will lose the connection which leads to BGP peer missing as well as network re-converging. For such problem, in the rest of the paper, we refer it as the satellite-terrestrial routing stability problem. We note that the fundamental cause of formation of IP level topology dynamic in the satellite-terrestrial routing stability problem is that IP addresses are designed to be bound to the satellites or the link interfaces of them. By such design, when a satellite moves, it will carry all the assigned IP addresses as well as the functions built upon them. This will result in massive signalling (i.e., the BGP Open/Notification/Update massage) exchanges between the e-BGP peers of the space and terrestrial segment and further leads to a complete BGP reconvergence within the whole integrated network which will significantly affect the stability of no only the space networks but also the terrestrial networks. In recent solution [14], the authors propose to establish the e-BGP connections using the loopback address, specifically, a ground station will constantly keep the e-BGP neighbourhood with a fixed satellite, even if the satellite has moved away and the ground station is attaching to a new satellite. However, such mechanism will significantly reduce the interaction efficiency between the two BGP peers, especially when the distance between the ground station and the satellite is considerably long. Not to mention that, if the new attaching satellite does not have the full routing information of the previous satellite (which can be the case when the two satellites belong to different ASes), the ground station will lose the e-BGP connection. Hence, such simple aggregation cannot fundamentally eliminate the problem.

\section{B. Geosynchronous Network Grid Addressing}

To tackle the satellite-terrestrial routing stability problem, we introduce the GNGA scheme. The main job of GNGA is to enable stable IP peers for the ground stations. To achieve that, we first decouple the IP addresses from the high mobility LEO satellites and construct a network framework in which the network addresses are assigned to a number of wellplanned spatial regions. In the rest of the paper, these spatial regions are referred to as the Geosynchronous Network Grids (GNGs). The GNGs are designed to be geosynchronous, such that from the perspective of the ground stations, the GNGs with the peering IP addresses are static despite the constellation behaviour of LEO satellites. Thereby, the complexity of integrating the terrestrial IP system with the high dynamic LEO satellite network is specifically confined within the space segment rather than being propagated into the legacy terrestrial infrastructures. Meanwhile, the incremental modifications of our design are from the space segments, which can be considered as implementation-friendly, because the IP deployment in space is still at the preliminary stage.

Figure 2 provides an illustration of the GNGA scheme. As shown in the figure, the GNGs are by logic the most basic routing unit at the network layer which can be regarded as the IP routers of the space segments, while physically, the GNGs are a set of planned spatial regions. To ensure the geo-synchronicity, the GNGs are defined with strict correspondence to the geography information ${ }^{1}$, e.g., the spatial region with 9/10th meridian east and 49/50th parallel north at $1000 \mathrm{~km}$ altitude is always defined as a GNG with statically allocated IP addresses. As such, a space network consists of a number of geo-static nodes (i.e., the GNGs) is logically created. In such network, the grids are geosynchronous while the LEO satellites are not. Thus, to enable the GNG to take the responsibility of the IP routers, we make the LEO satellites to consecutively support the IP function for the network grids. More specifically, when a satellite enters a network grid, it will have completed the updating of its IP configurations ${ }^{2}$ including the new binding of the IP addresses that have been statically associated with the GNG it enters, as well as the routing configurations should be pre-computed and cached at the LEO satellite side. Thanks to the highly-accurate predictable positions of LEO satellites, the activation of the corresponding IP configurations can be deterministically enforced. When the satellite is leaving the network grid, it will release the IP setting of the grid and adjust its settings based on the requirements of the next network grid.

Overall, in the context of GNGA, IP routers are the GNGs. If a ground station wishes to establish a BGP peer with the space segment, it should talk to a GNGs instead of any specific satellite.

\section{Design details of GNGA}

Putting aside the satellites, networking with GNGs has no essential differences to that with normal IP routers due to the geo-synchronicity. We follow the classic IP addressing and routing mechanism. That is to say, when a GNG is instantiated by the LEO satellite inside, it also follows the traditional forwarding table lookup process. It is desirable as welldeveloped IP applications/functions can be directly deployed in space and cause little impact on the current IP system, especially for the legacy terrestrial network segment. When taking the satellites into consideration, the major difference

\footnotetext{
${ }^{1}$ In addition to the longitude/latitude/altitude, time can also been taken as one of the dimensions

${ }^{2}$ In the rest of the paper, we use IP configuration and IP routing interchangeably.
} 


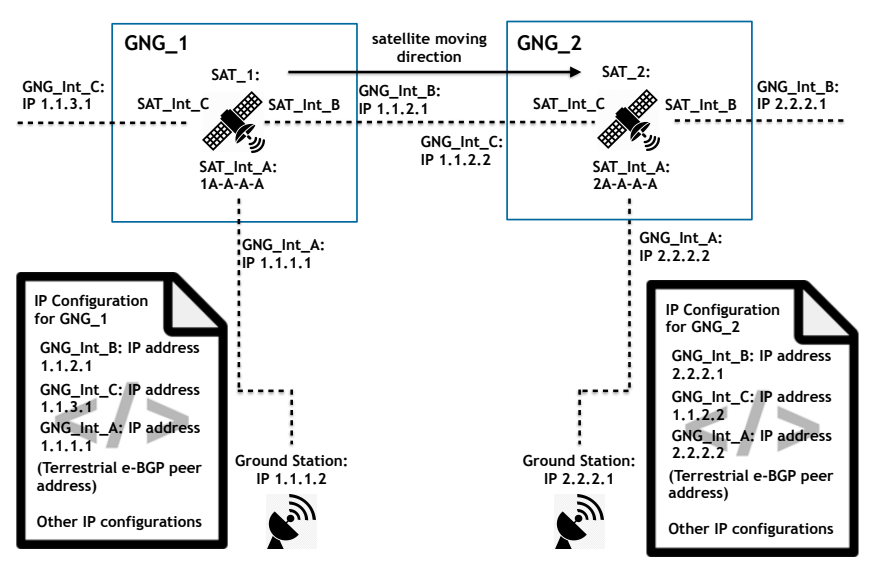

Fig. 3. Instantiating the GNGs with satellites.

is that the GNGs are instantiated by LEO satellites and the underlying satellite network would also need to provide the switching function for the network grids. In addition, since the satellites consecutively pass the GNGs to support the routing function, it is also important to ensure the satellites running the right IP settings at the right time when traversing a sequence of GNGs. These will be further discussed in the following features.

1. Instantiating the GNGs with satellites.

To instantiate a GNG, a satellite needs to activate the IP configuration of the grid it enters. Figure 3 shows an instance where GNG 1 and 2 are instantiated by satellite 1 and 2 , respectively. For illustration purposes, we only indicate in the figure two adjacent satellites belonging to the same constellation orbit. As shown in the figure, GNG 1 has 3 virtual interfaces and each virtual interface is assigned with an IP address. Satellite 1 in this case, should configure these IP addresses to its corresponding interfaces and especially the downlink interface towards ground stations i.e., 1A-A-A-A is mapping to 1.1.1.1 to establish the e-BGP connection with the terrestrial peer. Imagining after a while, satellite 1 is entering GNG 2 to take over the job of satellite 2, then it will need to configure its interfaces based on the configuration of GNG 2. In this case, 1A-A-A-A is now mapping to 2.2.2.2. If we consider GNG 2 to be the network grid shown in figure 2, then from the ground station's viewpoint, its space e-BGP peer is still 2.2.2.2 after the satellite handover event. Thus, as long as the satellite handover process is smooth enough, the e-BGP peer on the ground will not be aware of the change of the link. This aspect will be specifically tested in our future work.

2. Switching with the satellite network.

To facilitate the working of GNGA with regard to the knowledge of mapping operations of layer-3 IP address with the incoming satellite network interface, auxiliary layer 2 mechanisms need to be in place. And to leave the current satellite communication system unchanged to the largest extent, we adopt the satellite addressing and routing suit for the MAC layer. Without loss of generality, we assume each interface of a satellite has a physical address (also sees from figure 3). Accordingly, each IP address assigned to the GNG is to be temporarily held by a interface of the satellites. That is, each time when a LEO satellite moves into a network grid, it will map the IP addresses of the grid to its physical interface and advertise the mapping information to the network via the address resolution protocol (ARP). Then, the encapsulation and routing process will be identical to the classic paradigm. We note that, unlike the classic space-terrestrial network integration schemes where the ground station keeps tracking a specific satellite until its signal strength reaches a minimum threshold. While in the context of the GNGA scheme, the ground stations will point at a fixed angle and keep listening to the GNG instantiated by the passing LEO satellites. Once the ground station has caught the ARP information from the satellite, it will update its ARP cache with the prefix of the corresponding network grid and then it can establish normal IP sessions with the grid.

3. IP setting coordination.

In a classic network system, the IP setting is configured on physical devices in a one-off manner, hence each device normally possesses one setting at a time. However since in the GNGA scheme the setting is bounded to the logical GNGs that are periodically instantiated by passing-by LEO satellites, therefore for different grids, the settings will be different as well. This will require the satellites to mount the right setting when it reaches the corresponding network grid. This means, a satellite will need to periodically shift its setting during its mission. It is no doubt that the setting shift performance in this case plays an important role in the whole system. For the shifting method, we propose two designing options: the active shifting and the passive shifting. For the active option, each satellite carries the settings of all the grids during its mission and any of the GNG state update will be advertised to each satellite. The satellites therefore always keep the setting of each grid up to date and thereby can actively shift the settings based on the time-line or geography location. For the passive option, the satellites will rely on the predecessors to pass on the setting information. Specifically, when a satellite leaves a GNG, it will transmit its IP configuration to the next satellite. During this process, if the state of another GNG is changed and the update information has arrived before the GNG is instantiated by the new satellite, the satellite should redirect the updates to the new satellite. Hence, routing calculation is postponed to when the setting transmission is done. Obviously, Both options have their own advantages and disadvantages. The active option is easy to apply but at the price of low setting utilization. While the passive option provides a high setting utilization answer however it may experience longer convergence time.

\section{VALIDATION AND PRELIMINARY PERFORMANCE EVALUATION}

In the investigation, the proposed GNGA scheme is tested through emulation on a small scale topology which consists of 5 full-mashed terrestrial nodes and 3 GNGs. The GNGs are respectively instantiated by 3 satellites that share the same ring orbit. We define one of the terrestrial nodes as the ground 
TABLE I

EMULATION PARAMETERS

\begin{tabular}{ll}
\hline \multicolumn{1}{c}{ Parameter } & value \\
\hline Number of terrestrial nodes & 5 \\
Number of GNGs & 3 \\
Number of satellites & 3 \\
Number orbits & 1 \\
Number routing prefixes (terrestrial/space) & $10 / 3$ \\
\hline
\end{tabular}

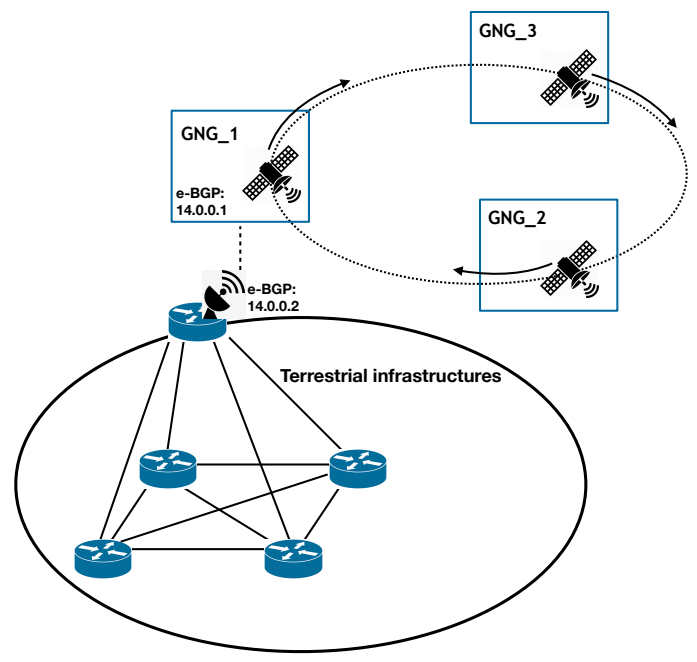

Fig. 4. Emulation topology

station for maintaining the BGP sessions with the GNG and we further assume the terrestrial and space networks are managed by different ASes. Without loss of generality, we assume the ground station always establishes the e-BGP connection with the closest GNG. For validation purpose, the number of routing prefixes are 10 from the terrestrial segment and 3 from the space segment. In regards to the satellite handovers, we define two types, namely, the smooth handover and the hard handover. For the smooth handover, the ground station will make the BGP connection with the new instantiating satellite before it releases the old one. In contrast, for the hard handover, the ground station releases the connection before the new connection is made. In summary, figure 4 provides a brief diagram of the emulation topology and the key parameters are listed in table I.

Figure 5 shows the change of network connectivity between the space and terrestrial segments during a satellite handover event. Here, connectivity being 1 represents the integrated network is converged and the space/terrestrial segment can perform normal communication with each other. To begin with, we perform hard handovers for the ground station. In this scenario, we introduce a 10-millisecond gap between the ground station making the connection with the new satellite and releasing the connection with the old satellite. As can be seen from the figure, directly applying BGP in the integration scheme requires on average 30 seconds to converge before the connectivity can be restored while GNGA recovers instantly when the new satellite has arrived. The result suggests that GNGA has a distinct advantage against the
BGP-straightforward integration scheme in terms of network convergence.

Next, we apply smooth handovers to circumvent the negative effect caused by the long BGP convergence time. As depicted in the figure, the improvement is immediate. In such scenario, satellite handover events do not disrupt the network connectivity for GNGA. However, we also notice that the BGP-straightforward integration scheme does not perform as seamless as GNGA. The cause of formation of such phenomenon is that, in the smooth handover scenario, there exists a short period that the ground station has two forwarding addresses respectively corresponding to the predecessor/successor satellites. Since for multiple external paths, a number of BGP instances by default prefer the earliest learned path (assuming other attributes are identical for these paths) due to the route-flapping prevention consideration [15], thus, the ground station will keep transmitting the data flow via the old path even after the new satellite has established the BGP session. As a result, when the ground station loses the connection with the previous satellite, the BGP processor will take an amount of time to switch the data flow to the new satellite.

Although the convergence issue of the BGP-straightforward integration scheme can be partly circumvented by adopting the smooth handover, it comes with prices. Figure 6 sheds some lights on understanding the trade-offs. As can be seen from the figure, with the hard handover the average number of update entries propagated into the terrestrial and space infrastructures are 9 and 97, respectively, which are considerably lower than the corresponding counterparts in the smooth handover scenario (i.e., 105 and 150, respectively). Thus, supporting the smooth handover for the BGP-straightforward integration scheme requires more computing resources to handle the updates. This is mainly attributed to the make-before-break feature of the smooth handover. Based on the feature, the link dynamic will be propagated through the redundancy link for path selection. For example, when the ground station has connected to the new satellite, its e-BGP session with the old satellite has not been released, in this case, the newly established link will be taken as a candidate path for the existing prefixes and the related updates will be propagated into the terrestrial/space network. Similar protocol behaviours will be performed again when the ground station loses the connection with the former satellite. In contrast, due to the delay between making and breaking the connection, there exists a transient period when the ground station has no connection with the satellites in the hard handover scenario. In this case, updates are only triggered by the connection/disconnection events rather than by the path selection events as well. As to the GNGA scheme, since the handover between each satellite is handled in layer 2, it will not trigger routing updates.

\section{SUMMARY AND FURTHER WORKS}

In this work-in-progress, a novel network integration scheme, namely, the Geosynchronous Network Grid Addressing is proposed for integrating the LEO satellite network 


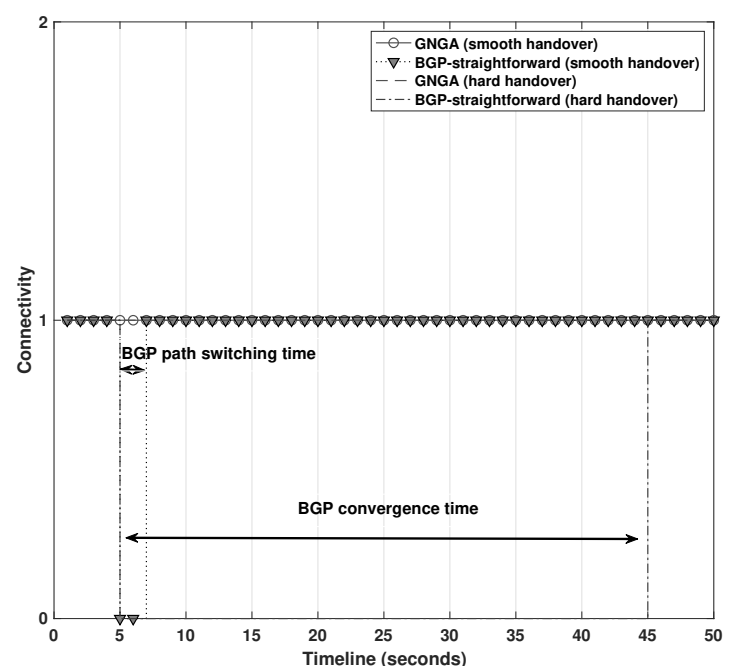

Fig. 5. The Space-Terrestrial connectivity performance of a single satellite handover event.

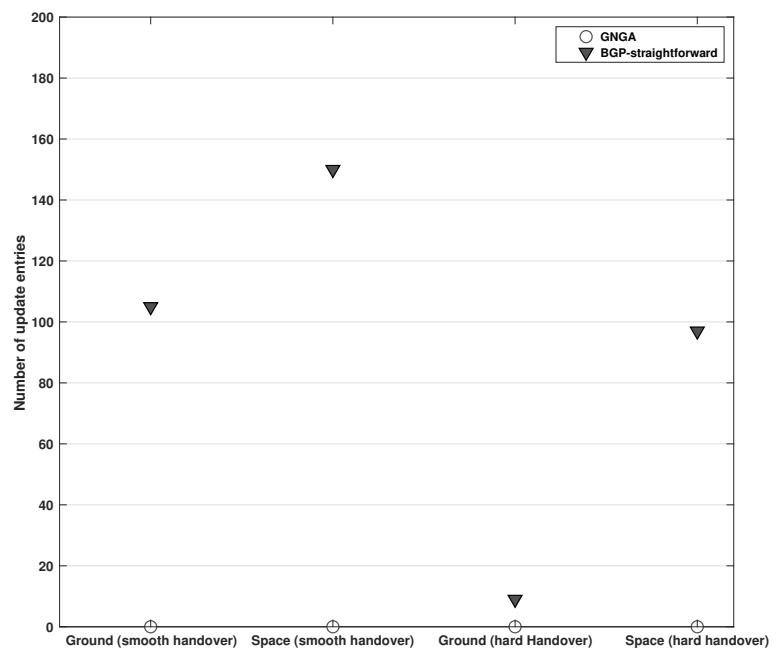

Fig. 6. The number of updates entries propagated into the space/terrestrial routing infrastructure.

into the legacy terrestrial network. Our design has distinct advantages in terms of protecting the terrestrial network from the integration impact. In addition, the proposed integration scheme is implementation friendly and the well-developed IP applications/functions can be directly deployed without any change.

The work is in progress and we are conducting further emulations to quantify the performance of the proposed GNGA scheme. Besides, We also plan to optimize the setting shifting methods to take a balance between the active and passive option.

\section{ACKNOWLEDGMENT}

This work is funded by Huawei and the authors would also like to acknowledge the support of University of Surrey's 5GIC (http://www.surrey.ac.uk/5gic) members for this work.

\section{REFERENCES}

[1] H. Yao, L. Wang, X. Wang, Z. Lu, and Y. Liu, "The space-terrestrial integrated network: An overview," IEEE Communications Magazine, vol. 56, no. 9, pp. 178-185, 2018.

[2] M. Handley, "Delay is not an option: Low latency routing in space," in Proceedings of the 17th ACM Workshop on Hot Topics in Networks, 2018, pp. 85-91.

[3] T. G. Griffin and B. J. Premore, "An experimental analysis of bgp convergence time," in Proceedings Ninth International Conference on Network Protocols. ICNP 2001. IEEE, 2001, pp. 53-61.

[4] B. Etefia, V. Swaminathan, J. Train, and J. Hant, "Emulating a spacebased router," in 2010 IEEE Aerospace Conference. IEEE, 2010, pp. $1-14$.

[5] Z. Yang, H. Li, Q. Wu, and J. Wu, "Analyzing and optimizing bgp stability in future space-based internet," in 2017 IEEE 36th International Performance Computing and Communications Conference (IPCCC). IEEE, 2017, pp. 1-8.

[6] D. Fischer, D. Basin, K. Eckstein, and T. Engel, "Predictable mobile routing for spacecraft networks," IEEE Transactions on Mobile Computing, vol. 12, no. 6, pp. 1174-1187, 2012.

[7] E. Ekici, I. F. Akyildiz, and M. D. Bender, "Datagram routing algorithm for leo satellite networks," in Proceedings IEEE INFOCOM 2000. Conference on Computer Communications. Nineteenth Annual Joint Conference of the IEEE Computer and Communications Societies (Cat. No. 00CH37064), vol. 2. IEEE, 2000, pp. 500-508.

[8] C. Chen, E. Ekici, and I. F. Akyildiz, "Satellite grouping and routing protocol for leo/meo satellite ip networks," in Proceedings of the 5th ACM international workshop on Wireless mobile multimedia, 2002, pp. $109-116$.

[9] Y. Yang, M. Xu, D. Wang, and Y. Wang, "Towards energy-efficient routing in satellite networks," IEEE Journal on Selected Areas in Communications, vol. 34, no. 12, pp. 3869-3886, 2016.

[10] T. ETSI, "102 292:"broadband satellite multimedia (bsm) services and architectures," Functional architecture for IP interworking with BSM networks.

[11] B. Di, L. Song, Y. Li, and H. V. Poor, "Ultra-dense leo: Integration of satellite access networks into $5 \mathrm{~g}$ and beyond," IEEE Wireless Communications, vol. 26, no. 2, pp. 62-69, 2019.

[12] G. Giuliari, T. Klenze, M. Legner, D. Basin, A. Perrig, and A. Singla, "Internet backbones in space," ACM SIGCOMM Computer Communication Review, vol. 50, no. 1, pp. 25-37, 2020.

[13] B. Trammell, J.-P. Smith, and A. Perrig, "Adding path awareness to the internet architecture," IEEE Internet Computing, vol. 22, no. 2, pp. 96-102, 2018

[14] Y. Zengyin, W. Qian, L. Hewu, and W. Jianping, "Ntd-bgp: An interdomain routing protocol for integrated terrestrial-satellite networks," Journal of Tsinghua University (Science and Technology), vol. 59, no. 7, pp. 512-522, 2019.

[15] C. Documentation, "Bgp best path selection algorithm," URL https://www.cisco.com/clen/us/support/docs/ip/border-gateway-protocolbgp/13753-25.html, 2006. 